\title{
Regulation of methanogenesis over introducing of humic compounds to biocatalytic systems with complicated content
}

Efremenko E. ${ }^{1,2}$, Senko O. ${ }^{1,2}$, Stepanov N. ${ }^{1,2}$, Volikov A.B. ${ }^{1}$, Mareev N.V. ${ }^{1}$, Perminova I.V. ${ }^{1}$

${ }^{1}$ Lomonosov Moscow State University, Moscow, Russia, elena_efremenko@gmail.com

${ }^{2}$ Emanuel Institute of biochemical physics RAS, Moscow, Russia

doi: 10.36291/HIT.2019.efremenko.013

Landfills of solid household waste pose a serious long-term risk to the environment and public health. This is due to the active production of landfill gas, which mainly contains $\mathrm{CH}_{4}$ and $\mathrm{CO}_{2}$ with a low addition of $\mathrm{H}_{2} \mathrm{~S}, \mathrm{H}_{2}$, and $\mathrm{NH}_{3}$. For this reason, in many developed countries, special procedures are used to minimize landfill gas emission. The search for new solutions to reduce the intensity of landfill gas production and the proportion of $\mathrm{CH}_{4}$ in its composition is highly relevant.

$\mathrm{CH}_{4}$ is formed as a result of the metabolic activity of methanogenic microbial consortia that are formed spontaneously under natural conditions and consist of hydrolytic and acetogenic bacteria as well as methanogenic archaea [1].

Recently, the inhibition of the methanogenic activity of anaerobic consortia by various humic compounds (HCs) containing various functional (carboxyl, phenolic, hydroxyl, etc.) groups, has been actively studied [2]. At the same time, it should be noted that under real conditions (in landfills), microorganisms involved in the formation of biogas are in a stable state due to both the formation of a stable consortia, high cell density, and immobilization (sorption predominantly) on various materials. In this regard, it seemed appropriate to study the effect of HCs on the methanogenic consortia and the methanogenesis process itself in the presence of naturally and artificially immobilized forms of anaerobic microorganisms [1].

The purpose of this work was to evaluate the activity of various natural methanogenic consortia using the bioluminescent luciferin-luciferase method of ATP determination and to compare this method with the traditionally used approach based on studying the characteristics of methanogenesis: the process efficiency and the methane content in the accumulated biogas. In particular, the new technique was applied to assess the effect of different concentrations of HCs. This approach was used to analyze the effect of introducing potassium humate and fulvic acids (1-10 g/L) into media with different methanogenic consortia producing biogas.

The increase in the concentration of potassium humate led to a decrease in the overall energy status of the cells and reduced methanogenesis efficiency. However, fulvic acids introduced into the media stimulated methanogenesis in half of the tested consortia, which was accompanied by an increase in ATP concentration in cell samples. So, a positive correlation between the metabolic activity of cells in biogas formation and the concentration of ATP was observed. ATP concentration control appears to be an attractive tool for finding compounds that suppress methanogenesis in landfills.

Acknowledgements. This research was funded by the Russian Foundation for Basic Research (Grant No. 18-29-25065)

References

1. Senko et al. // Catalysts. 2019. 9(4):326.

2. Stepanov et al. // Sustainability. 2019. 11:3158-11. 